\title{
Bridge to success and inclusivity
}

\author{
The American Physical Society's Bridge Program increases the output of doctorates \\ awarded to underrepresented graduate students in the physical sciences.
}

\section{By Roy Clarke and Çagliyan Kurdak}

\begin{abstract}
$\mathrm{A}^{\mathrm{t}}$ some point in our professional lives, we all have a bridge to cross. More often than not, an inspiring mentor has been critical in helping us along the way. This is most true when undergraduate students aspire to transition into graduate studies from being passive receptors of knowledge into full-fledged research innovators. For many, this is a daunting challenge beset with hurdles, not all of them relevant to the eventual success as a creator of knowledge. In this article, we discuss some initiatives under way to address the lack of diversity in the science, technology, engineering, and mathematics (STEM) fields. Our aim is to touch on some of the most recent developments that are especially of interest to the Materials Research Society (MRS) and its members.
\end{abstract}

\section{Challenges}

The challenges encountered by underrepresented and nontraditional students

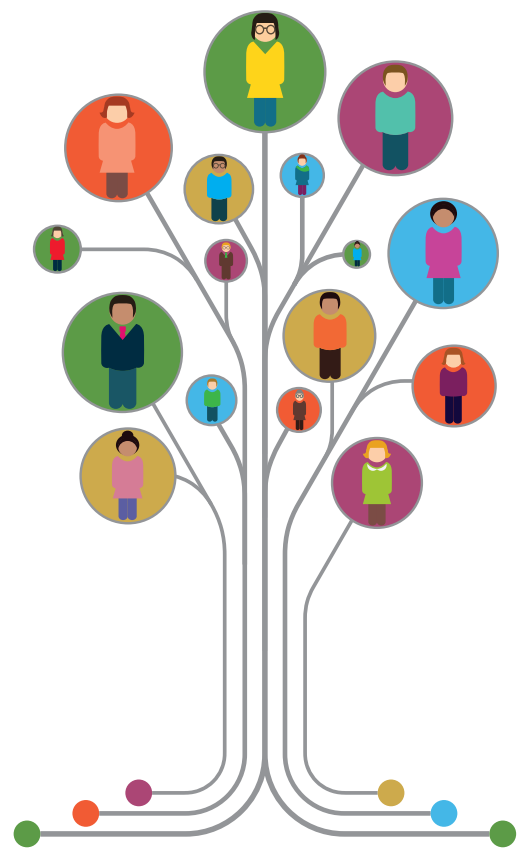

(e.g., those in the workforce seeking additional qualifications) can be particularly frustrating, especially given the persistent and widespread "gatekeeper" culture that continues to pervade physical sciences and engineering. The American Physical Society's (APS) Bridge Program, initiated in 2012, seeks to increase the output of doctorates awarded to graduate students from underrepresented groups in the physical sciences in the United States by promoting innovative approaches to recruiting and nurturing students. While many scientific fields struggle with diversity, physics especially is among the least diverse. This situation is only marginally better for materials science and engineering. (See the Diversity in Materials Science and Engineering Series in the 2018 MRS Bulletin at www.mrs.org/bulletin/diversity.) According to APS, approximately $12 \%$ of all students receiving bachelor's degrees in physics in the United States are from underrepresented racial and ethnic minority backgrounds, which includes African Americans, Hispanic and Latin Americans, Indigenous Americans, and Pacific Islanders, whereas only $7 \%$ of $\mathrm{PhD}$ students come from such groups. With the goal of essentially doubling the number of physics doctoral students from these communities at US universities during the next few years, the APS Bridge Program focuses on improving the mentoring of undergraduates, modifying admission practices for graduates, recruiting students who might not otherwise have considered entering graduate studies, and providing ongoing mentoring for such students.

\section{Building bridges}

The genesis of this bridge-building approach can be traced back to the establishment of a radical new interdisciplinary doctoral program at the University of Michigan (UM)-Ann Arbor, in 1987. The UM Applied Physics (AP) Program was set up with the express purpose of enhancing research and graduate training collaborations between the Physics Department and various departments of the Engineering College, Materials Science and Engineering being a case in point as one of the most cross-disciplinary. As the program developed, many additional interdisciplinary connections were made across other university units, including the Medical School, Environmental Sciences, and the Schools of Public Health and Public Policy - in fact, any place where the application of physics could have an impact that befits the central, integrative role of physics across all of the sciences and engineering. The program now brings together more than 150 professors from more than a dozen departments and includes close to 100 enrolled doctoral students at UM.

The unusual scientific variety of the UM AP program, in terms of both people and scientific fields, has diversity, equity, and inclusion built into its DNA. The program's uniquely broad structure is a major advantage for underrepresented students and those from nontraditional backgrounds, because it provides students with more options and helps them discover their individual pathway to success. This "bandwidth" offers a vastly increased number of channels through which a student's entry into a career in research can be facilitated. Moreover, it helps to counter the perception, particularly among students from underrepresented communities, that a lack of opportunities exists for career advancement in STEM fields. It is tragic that the United States has such 
a large pool of underutilized and unrealized STEM talent, while the system is crying out for a more skilled workforce in our knowledge-based economy. Getting more players "off the bench and into the game," as Shirley Malcom, head of the Education and Human Resources Programs for the American Association for the Advancement of Science, so aptly put it, is one of the greatest challenges. This is something the AP program, with its mantra of "building bridges not walls," has sought to tackle with some success over the years.

On average, $17 \%$ of physics $\mathrm{PhD}$ degrees are awarded to women in the United States. At UM, however, $29 \%$ of all $\mathrm{AP} \mathrm{PhD}$ degrees are awarded to women, and $23 \%$ are awarded to minorities. This success, in part, stems from providing a pathway from undergraduate studies into graduate work at the master's level, and hence into doctoral studies, via the ImesMoore Fellows Program, named for Elmer Samuel Imes, the second African American to be awarded a $\mathrm{PhD}$ in physics, and Willie Hobbs Moore, the first African American woman to receive a $\mathrm{PhD}$ in physics, both from UM. The Imes-Moore program was spearheaded by one of the co-authors of this article, Çagliyan Kurdak, the current director of the AP program. Unlike other bridge programs in physics, where students generally enter a $\mathrm{PhD}$ program at a different institution, the Imes-Moore program aims to fully integrate the bridge students into a $\mathrm{PhD}$ program at $\mathrm{UM}$.

One advantage UM holds over most of its peer institutions is that it has a significant critical mass of underrepresented students in the $\mathrm{PhD}$ program who are willing to serve as peer mentors for incoming students. During the last eight years, 21 students have gone through the ImesMoore program at UM, and 14 of these students have successfully transitioned into the $\mathrm{PhD}$ program. UM, together with bridge sites at Fisk-Vanderbilt universities, Columbia University, and the Massachusetts Institute of Technology, plays an advisory role in the launching of the APS Bridge Program. The best practices first implemented in the AP program had a direct effect on many of the subsequent bridge sites that were launched though the APS Bridge Program.
What are the lessons learned and how do these feed into initiatives, such as the APS Bridge Program? Many explanations have been suggested regarding the underrepresentation of women and minorities in physical sciences and STEM disciplines overall. Traditionally, many physics departments tend to maintain a somewhat elitist "sink or swim" attitude, which is unwelcoming to students from nontraditional backgrounds. Standardized metrics used to admit physics students, such as the GRE subject test, are often rigidly applied, overlooking the overall potential for creativity. A more holistic set of admissions criteria needs gram has advocated from the outset, and which is now being widely recognized and implemented.

Qualifying examinations are another area of contention. The traditional written test often presents a barrier for students in interdisciplinary programs, where not everyone has the same academic background, and for talented students from smaller undergraduate programs that cannot field a broad range of courses. Better then to organize the qualifier process as a diagnostic to identify the gaps in a student's preparation and the additional courses required to fill them, information that is more efficiently gained in an interview setting rather than a formal written exam. This enters to the heart of the bridge philosophy: recognizing that in a highly interdisciplinary research world, one size most definitely does not fit all.

\section{Path to success}

How then, can bridge programs help with student success, particularly the success of female, minority, and nontraditional $\mathrm{PhD}$ students? Put into context, the success rate in many physics doctoral programs is below $50 \%$, which is a shocking waste of resources and talent. The AP program has been able to maintain a graduation rate of more than $90 \%$ by establishing support mechanisms, such as peer mentoring, early entry into research, academic diagnostics as mentioned, formal progress tracking, and opportunities for social and professional interactions among students and faculty. Most importantly, best practices include access to a broad range of research opportunities across disciplines, a feature that physics embraces well.

One of the barriers to offering early entry into research is that research group leaders are often reluctant to provide financial support (research assistantships) to students who are taking a substantial course load and cannot fully contribute to research. It is therefore essential that fellowship funds are available in the early, pre-candidate years of graduate studies. This is one of the vital components of the bridge program, and fortunately several agencies (e.g., National Science Foundation [NSF], US Department of Defense, US Department of Energy) and foundations (e.g., Sloan) have stepped up their fellowship programs to ensure funds are available for pre-candidate support. Fellowship application workshops are also a good way to inform students about the process of applying for these funds.

\section{Initiatives}

Recognizing the great potential of bridge programs and the need for a more concerted effort, five leading US scientific societies have come together to launch a new program to boost the diversity of the graduate student body in STEM areas. The Inclusive Graduate Education Network (IGEN), coordinated by NSF's 
INCLUDES program, will receive $\$ 10$ million over the next five years to improve diversity in astronomy, chemistry, geoscience, materials science, engineering, and physics. The five partner societies are MRS, the American Astronomical Society, the American Chemical Society, the American Geophysical Union, and APS.

In November 2018, MRS participated in an IGEN Alliance Management Team (AMT) kick-off meeting held in conjunction with the APS National Mentoring Community and Bridge Program national meeting. During the meeting, the NSF INCLUDES Alliance and IGEN partners discussed overall program goals as well as individual organizational goals for expanding bridge programs into their respective disciplines. This provided a great opportunity for IGEN AMT members to interact with underrepresented students and faculty participating in bridge programs and to learn about their experiences. It also served as a forum for the AMT to become acquainted, to explore how to continue building the alliance, and to forge partnerships. For MRS, 2019 will be a year of recruiting volunteers, planning IGEN activities, and communicating with the materials community and underrepresented students about inclusive practices and research, as well as Bridge Program opportunities.
The initiatives we are currently seeing at the local level, as well as on a national scale, are a recognition of the importance of this issue, and should provide a strong platform from which to enhance US leadership and excellence in STEM subjects by broadening participation in those areas. If you are a MRS member with an interest in helping lay the groundwork for a Bridge Program in the materials science and engineering discipline, contact Michele Feder, feder@mrs.org.

This article is dedicated to MIT professor and mentor, Mildred (Millie) Dresselhaus (1930-2017).

\section{MIR|S FALL MEETING \& EXHIBIT}

Thank you to the following organizations for their 2018 MRS Fall Meeting symposium support:

ACS Division of Inorganic Chemistry

ACS Energy Letters | ACS Publications

Acta Biomaterialia (Acta Materialia Inc.) | Elsevier

Acuitive Technologies, Inc.

Advanced Photon Source at Argonne National Laboratory

Allevi, Inc.

American Elements

Angstrom Engineering Inc.

Angstrom Thin Film Technologies LLC

Applied Materials

Arios Ltd.

BAE Systems

Binghamton University, Center for Advanced Microelectronics Manufacturing, New York Node-NextFlex Manufacturing USA

Biofabrication | IOP Publishing

Bio-Logic USA, LLC

Carat Systems

The Center for Advanced Regenerative

Engineering (CARE), Northwestern University

Chem | Cell Press

Chemistry of Materials | ACS Publications

Cline Innovations, LLC

Cornes Technologies, Seki Diamond Systems

Crystal Growth \& Design | ACS Publications

Daicel Corporation

Delaware Diamond Knives, LLC

DiamFab

EDP Corporation

Forschungszentrum Jülich $\mathrm{GmbH}$

Fraunhofer USA

GE Global Research

Georgia Institute of Technology, Institute for

Materials

Goodfellow Corporation

Gotion Inc.
Heraeus

Hiden Isochema Ltd.

Hitachi High Technologies America, Inc.

INDEED Network

Inorganic Chemistry | ACS Publications

International Centre for Diffraction Data (ICDD) IQE plc

JEOL USA, Inc.

Joule | Cell Press

Journal of Physics and Chemistry of Solids

Elsevier

Journal of Solid State Chemistry | Elsevier

Keithley, A Tektronix Company

King Abdullah University of Science and

Technology

Kyoto University

LC Technology Solutions Inc.

Linseis Inc.

Los Alamos National Laboratory

Malvern Panalytical

Materials Today | Elsevier

Materials Today Bio | Elsevier

Metal Technology Co., Ltd.

Michigan Technological University,

Henes Center for Quantum Phenomena

Microwave Enterprises

MilliporeSigma

Montanuniversitaet Leoben

MTI Corporation

Nanoscale | Royal Society of Chemistry

National Institute of Standards and Technology

National Renewable Energy Laboratory

National Science Foundation

National Science Foundation, Division of

Materials Research

Nature Energy | Springer Nature

NETZSCH Instruments North America, LLC
New Diamond Technology

Novel Crystal Technology, Inc.

NVision Imaging Technologies

Pacific Northwest National Laboratory

Photon Etc.

Physical Review Materials | American Physical Society

Plasmability, LLC

Protochips, Inc.

Rigaku

Royal Society of Chemistry

Science and Technology of Advanced Materials

National Institute for Materials Science

Science Robotics | AAAS

Sinton Instruments

Solar RRL | Wiley

Spin Phenomena Interdisciplinary Center (SPICE)

Springer Nature

Sunano Group Limited

Thermo-Calc Software Inc.

Toyota

The UK Skyrmion Project

University of Illinois at Urbana-Champaign (Coordinated Sciences Lab and Materials Science \& Engineering)

University of Massachusetts, Institute for Hierarchical Manufacturing, Massachusetts Node-NextFlex Manufacturing USA

University of Michigan, Department of

Mechanical Engineering

The University of Texas at Dallas, Alan G. MacDiarmid NanoTech Institute

The University of Texas at Dallas, School of Natural Sciences and Mathematics

The University of Utah, Department of Chemistry

Wiley-VCH Verlag GmbH \& Co. KGaA

ZEON Corporation 